\title{
Kongressbericht EBCC 7, 2010, Barcelona
}

\author{
Florian Fitzal \\ Abteilung für Allgemeinchirurgie, Brustgesundheitszentrum, Medizinische Universität Wien, Österreich
}

Der Europäische Brustkrebskongress in Barcelona stand im Zeichen von Lehrveranstaltungen; obwohl einige nicht publizierte Studien als Poster vorgestellt wurden, haben es nur wenige zu einer Posterdiskussionen mit eingeladenem Vortrag geschafft. Dieser Bericht soll jedoch weniger auf die Lehrveranstaltungen, als auf einzelne Studien eingehen, deren Themen mir persönlich wichtig erschienen, auch wenn die Studiendaten nicht unbedingt auf hoher Evidenz basierten.

Die Zusammenfassung stellt keinen Anspruch auf Vollständigkeit und kann nur als subjektive Berichterstattung gesehen werden. Publiziert sind die Abstracts im Eur J Cancer Vol 8 (3) ISSN 1359-6349.

\section{Lokale Therapie: Chirurgie}

Der wichtige Einfluss der lokalen Therapie auf das Lokalrezidiv ist hinlänglich bekannt. Die EBCTCG Übersichtsdaten zeigen auch, dass das Lokalrezidiv einen signifikanten Einfluss auf das Gesamtüberleben hat. Dies wurde durch eine gepoolte Analyse von prospektiven Daten der EORTC Datenbank bestätigt. Die Studie von van der Hage et al. (Abstract 106) mit über 7000 Frauen und einer Nachbeobachtungszeit von 10 Jahren zeigte, dass das Lokalrezidiv-freie Überleben in einer multiplen Varianzanalyse den stärksten unabhängigen Faktor für das Überleben darstellte (Hazard Ratio (HR) 5,07).

Es konnte gezeigt werden, dass die Wartezeit von Diagnose bis zur Operation auf Grund vermehrter Etablierung präoperativer Biopsien und interdisziplinärer Tumorboards wieder ansteigt verglichen mit einem historischen Krankengut. Min et al. zeigten, dass eine Wartezeit von über 28 Tagen - verglichen mit unter 28 Tagen - aber keine Auswirkungen bezüglich Tumorstadium und Anwendung einer Mastektomie haben dürfte (Abstract 132).

Im Zeitalter der onkoplastischen Chirurgie konnte Ballardini et al. zeigen, dass eine brustwarzenerhaltene Mastektomie mit Sofortrekonstruktion eine onkologisch sichere Variante ist, solange man den retromammillären Bereich histologisch als unauffällig klassifizieren konnte. Bei 175 Präparaten traten nach 41 Monaten 3,4\% Lokalrezidive auf, wobei nur eines im Sinne eines Pagets in der Mamilla zu finden war (Abstract 232).
Um die Diskussion des Resektionsrandes ging es in einer Lehrveranstaltung, der Giuseppe Viale und Michael Gnant vorsaßen. 2 Posterarbeiten demonstrierten, dass für ein invasives Karzinom ein Resektionsrand von $1 \mathrm{~mm}$ ausreichen dürfte ( $\mathrm{n}=550$, Abstract 256), für ein Ductales Carcinoma in situ (DCIS) allerdings $5 \mathrm{~mm}$ als Mindestabstand zu sehen sind ( $\mathrm{n}=293$, Abstract 262). Ein kürzlich publizierter Konsensus besagt, dass Karzinomzellen eines invasiven Karzinoms die Tuschemarkierung nicht erreichen dürfen, für das DCIS sollte der Resektionsrand nach einer 2009 im JCO publizierten Studie von Monica Morrow $>2 \mathrm{~mm}$ betragen.

\section{Lokale Therapie: Radiotherapie}

Reitsamer stellte die 10-Jahres-Daten der Salzburger Landesklinik von Frauen da, welche mit einer intraoperativen BoostTherapie gefolgt von einer Ganzbrustbestrahlung therapiert wurden (Abstract 239). Sein Vergleich mit der herkömmlichen Brachytherapie konnte zeigen, dass das Lokalrezidiv inklusive Axillarezidiv in der intraoperativen Boost-Gruppe nur knapp nicht signifikant seltener war (3\% versus $7 \%, p=0,06)$, wohingegen echte Lokalrezidive in der intraoperativen Gruppe signifikant seltener waren $(0,7 \%$ versus $5 \%, p=0,016)$. Rezidivfreies und Gesamtüberleben konnten nicht verbessert werden. Wird eine Strahlentherapie nach brusterhaltender Operation unterlassen, so erhöht dies bei Patientinnen mit einem T1/2 N0 Karzinom unter Tamoxifen das Lokalrezidivrisiko signifikant (Abstract 231; $\mathrm{n}=769$, 10-Jahres-Daten $13 \%$ versus 5\%).

\section{Lymphinvasion}

Die Daten von ONCOPOOL konnten zeigen, dass die lymphovaskuläre Invasion ein Lymphknotenstatus-unabhängiger schlechter prognostischer Marker für das Überleben ist (Abstract 125), wohingegen dieser Faktor scheinbar weder nach Brusterhaltung, noch nach Mastektomie einen signifikanten Einfluss für das Lokalrezidiv-freie Überleben darstellte (Abstract 163).

\section{KARGER}

Fax +497614520714

Information@Karger.de

www.karger.com
(C) 2010 S. Karger GmbH, Freiburg

Accessible online at:

www.karger.com/brc 


\section{Sentinel-Lymphknoten}

Die Mikrometastasen $(<0,2 \mathrm{~mm})$ haben auch bei diesem Kongress einen großen Teil der Poster für sich beansprucht. Eine niederländische Analyse von über 400 Frauen mit 6\% Mikrometastasenanteil konnte keinen Einfluss von pN1mic auf das Überleben entdecken (Abstract 235). Sola et al. berichten über eine prospektive Studie an 248 Frauen mit einer Mikrometastase nach Sentinel-Biopsie. In der Gruppe mit axillärer Dissektion $(n=113)$ hatten $13 \%$ einen weiteren positiven Lymphknoten. Die Nachbeobachtungszeit ist noch nicht erreicht, sodass noch keine Aussage über die Häufigkeit von Lokalrezidiven getroffen werden konnte (Abstract 307).

Es ist immer wieder die Frage, ab welcher Größe eines DCIS eine Sentinel-Biospie durchgeführt werden soll. Mentrak et al. konnten bei 285 DCIS-Patientinnen zeigen, dass 7\% einen positiven Sentinel-Lymphknoten hatten. Alle zeigten im endgültigen Präparat Zeichen einer Invasion. In 90\% der Fälle war die Mikroverkalkung über $4 \mathrm{~cm}$ groß. 70\% hatten ein schlecht differenziertes Grading. Aus diesem Grund empfehlen die Autoren, dass alle präoperativ bioptisch verifizierten DCIS G3 mit einem Mikrokalkdurchmesser von über $4 \mathrm{~cm}$ einer primären Sentinel-Biopsie zugeführt werden sollten (Abstract 309).

\section{Adjuvante Therapie}

Hasenbrug et al. konnten zeigen, dass ein Switch von Tamoxifen auf Exemestan ähnlich wirksam ist, das Rezidiv-freie Überleben zu verbessern, wie 5 Jahre Exemestan (Abstract 15). In der TEAM-Studie wurden über 9000 Frauen randomisiert. Die primär berichtete Verbesserung des Rezidiv-freien Überlebens durch Exemestan alleine nach 2,7 Jahren konnten nun nach 5 Jahren nicht mehr nachgewiesen werden.

\section{Neoadjuvante Therapie}

Ein wichtiges Thema ist die Frage nach einem prädiktiven Marker für das pathologische komplette Ansprechen auf eine Chemotherapie (pCR), um Patientinnen zu identifizieren, welche in jedem Fall von einer neoadjuvanten Chemotherapie profitieren (besser wäre es, Tumore zu kennen, welche zu $100 \%$ nicht ansprechen - bis jetzt ist das aber noch nicht gelungen). In diesem Sinne konnten Steger et al. durch Analyse der ABCSG-24-Studie zeigen, dass das Tumorstadium, das Grading, der Hormonrezeptorstatus und die Gabe von Capecitabin zusätzlich zum Standardregime unabhängige prognostische Faktoren für das Ansprechen auf die Chemotherapie waren (Abstract 8, poster discussion). Eine Reduktion des HER2-Serumspiegels unter $10 \mathrm{ng} / \mathrm{ml}$ war ebenfalls prädiktiv für das Ansprechen auf eine Chemotherapie (Witzel et al., Abstract 12, poster discussion). Lips et al. konnten zeigen, dass eine Mutation im BRCA1- und -2-Gen mit einem besse- ren Ansprechen auf eine neoadjuvante Therapie korreliert (Abstract 10, poster discussion) und eine Analyse unserer Patienten der letzten Jahre $(\mathrm{n}=308)$ mit einer Nachbeobachtung von 60 Monaten zeigte, dass der Tumortyp (ductal), der Rezeptorstatus (pos) und der HER2-Status (pos) signifikante unabhängige prädiktive Marker für eine pCR waren (Abstract 17). Cho et al. zeigten, dass der HER2-Status, der Rezeptorstatus und eine klinisch negative Axilla unabhängige Prädiktoren für das Ansprechen waren (Abstract 29).

Semigalzov hat wieder eine endokrine neoadjuvante Studie präsentiert (Abstract 9, poster discussion) und konnte zeigen, dass bei postmenopausalen Patientinnen mit einem Rezeptorpositiven Karzinom das Ansprechen auf eine endokrine Therapie (Aromataseinhibitor) mit dem auf eine Chemotherapie vergleichbar war. Das Lokal- und Fernmetastasen-freie Überleben war in beiden Gruppen nach einer Beobachtungszeit von über 5 Jahren gleich. Die Zugabe von Fulvestrant zu einem Aromataseinhibitor über 21 Tage als neoadjuvante Therapie erbrachte keine höhere Proliferationsreduktion (Robertson, Abstract 18).

Chirurgisch konnte unsere Gruppe zeigen, dass die brusterhaltende Operation nach Tumordownsizing ein onkologisch sicheres Verfahren darstellt. Diese retrospektive Analyse von 308 Frauen mit einer Nachbeobachtung von 60 Monaten unterstützt die letzte Metaanalyse zu diesem Thema. Wichtig ist, dass bei Patientinnen, welche primär eine Mastektomie benötigen und kein Tumordownsizing erfahren, keine Brusterhaltung versucht werden sollte. Das onkologische Ergebnis ist bei diesen Patientinnen durch eine brusterhaltende Therapie signifikant schlechter als nach einer Mastektomie (Abstract 26).

\section{Lebensqualität/Psychologie}

Eine Studie des Karolinska Institutes zeigte, dass Selbsthilfegruppen einen signifikant positiven Effekt auf Ängste von Patienten ausüben (Abstract 76). Das Selbstbewusstsein von Patientinnen wurde in einer prospektiven Studie nicht durch eine brusterhaltende Operation oder durch die Gabe von Chemotherapie beeinflusst. Die Nebenwirkungen wie Müdigkeit und depressive Symptome waren aber unabhängige Faktoren für eine schlechtere Lebensqualität (Abstract 78). Unsere eigenen Analyse zum Thema Lebensqualität konnten zeigen, dass nicht die Symmetrie, sondern die Form der Brust an sich das Selbstbildnis und die Lebensqualität durch den Einsatz onkoplastischer Techniken verbessert (Abstract 86).

\section{Metastasiertes Mammakarzinom}

Ribeiro konnte an Hand von 25 Patientinnen zeigen, dass die Tumorbiologie zwischen Primärkarzinom und Metastasen in 46-60\% der Fälle unterschiedlich sein kann (Abstract 195). Auf diese Tatsache wird in der palliativen Therapie immer mehr Rücksicht genommen, genaue Studien dazu fehlen aber. 